\title{
Téoros
}

Revue de recherche en tourisme

\section{La mise en marché dans le réseau de distribution touristique}

\section{Jean-Marc Descôteaux}

Volume 5, numéro 2, juillet 1986

Tourisme, fêtes et événements

URI : https://id.erudit.org/iderudit/1080578ar

DOI : https://doi.org/10.7202/1080578ar

Aller au sommaire du numéro

Éditeur(s)

Université du Québec à Montréal

ISSN

0712-8657 (imprimé)

1923-2705 (numérique)

Découvrir la revue

Citer cet article

Descôteaux, J.-M. (1986). La mise en marché dans le réseau de distribution

touristique. Téoros, 5(2), 33-35. https://doi.org/10.7202/1080578ar d'utilisation que vous pouvez consulter en ligne.

https://apropos.erudit.org/fr/usagers/politique-dutilisation/ 


\title{
La mise en marché dans le réseau de distribution touristique
}

\author{
par Jean-Marc Descôteaux *
}

Depuis quelques années, la majorité des intervenants touristiques doivent tenir compte d'une nouvelle dynamique dans la la promotion du tourisme au Québec: celle des événements majeurs (artistiques, sportifs, populaires).

L'industrie touristique québécoise avait déjà dũ dans un passé récent adapter ses infrastructures (hôtellerie, restauration, accueil) au phénomène des congrès, des colloques, des réunions d'affaires et ce, principalement à Montréal et à Québec. Les événements majeurs, artistiques ou sportifs, -festivals, expositions, Grand Prix automobile, etc- requièrent cependant une approche différente ainsi qu'une collaboration entre le monde du tourisme et celui des arts. Une grande souplesse et une véritable volonté de collaborer doivent se retrouver tant chez les organisateurs de ces événements que chez les partenaires touristiques.

\section{Le pourquoi \\ de cette collaboration}

Si vous êtes un intervenant oeuvrant dans les milieux artistiques, culturels ou sportifs et que vous songez à collaborer avec des entreprises à vocation touristique, votre action doit viser des objectifs spécifiques quant à la tenue de l'événement. Ces objectifs peuvent être de deux ordres: l'aide technique à la réalisation de l'événement (ou soutien logistique) et la promotion de

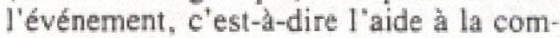
mercialisation par la création de forfaits culturels et touristiques. Le contact entre les deux secteurs devrait également susciter une meilleure compréhension de la réalité de ces deux mondes, à la fois si peu et très éloignés l'un de l'autre. Le premier contact devrait donc permettre au partenaire touristique envisagét! de savoir si on attend de lui la prise en charge logistique ou sa collaboration à la promotion de l'événement.

\footnotetext{
- Jeen-Marc Descóteaux est chargé de cours au module de gestion et intervention touristiques de I'UQAM. En 1985. il a ette adjoint aux communications et a la promotion touristique au Festival de thedtre des Amé ristiques ot en 1986, il a éte coobrdonnateur a l'accueil au Festival international de mimes de Montréal.
}

L'aide technique à la réalisation de l'événement Vous faites partie du comité organisateur d'un festival artistique ou d'une manifestation sportive; il vous faudra songer rapidement, quasi dès les dates officielles de l'eveúnement déterminées, à vous mettre en contact avec des fournisseurs de services à caractère touristique. Ainsi l'une des premières préoccupations dudit comité sera de signer une entente avec un ou des hóteliers afin d'assurer l'hébergement des participants et des festivaliers étrangers ou en provenance d'une région autre que celle où se déroulera l'événement. Cette démarche sera particulièrement importante si la manifestation se déroule dans une grande ville. à une période touristique de pointe (entre mai et septembre) et si vous prévoyez un nombre élevé de participants ( 500 et plus). Mais aurez-vous le temps de visiter plusieurs hôtels de différentes catégories, de les comparer, de négocier des tarifs préférentiels avec les départements de ventes respectifs et obtenir ainsi des tarifs "garantis" jusqu'aux dates de l'événement?... La recherche de cet hôte officiel de votre manifestation s'accompagne régulièrement de la recherche de restaurants, bars, centres de conditionnement physique, salles de réunion, etc. Certains hôtels commencent à s'ouvrir à la nouvelle réalité de ces évênements qui prennent maintenant d'assaut la métropole - particulièrement en saison estivale - et dédident d'associer le nom de leur établissement à la promotion de l'événement.

En cours des préparatifs inhérents a l'événement, wous devrez peut-être retenir les services de conférenciers, d'invités spéciaux, de compagnies artistiques ou d'équipes sportives venant de l'etranger. Une fois la liste connue, vous contacterez probablement un(des) transporteur(s) aérien(s) ou terrestre(s) ou encore une agence de voyages. En confiant le travail très technique de la négociation des billets d'avion et des tarifs à une agence de voyages, vous n'économiserez peut-être pas beaucoup d'argent car en général il s'agira de billets en première classe, plein tarif, et prépayés mais il y aura une économie de temps et d'éner- gie en raison des personnels qualifiés des agences de voyages pour effectuer ce type de travail.

Aimeriez-vous cependant vous assurer les services d'un transporeur officiel aérien? Air Canada et Canadien Pacifique, transporteurs nationaux, sont en général heureux et ouverts à l'idée de s'associer à des manifestations d'envergure. Ils peuvent faire bénéficier vos clientèles de réductions substantielles des tarifs et de services connexes comme une réservation facilitée de sièges, le pré-enregistrement, le transport de marchandises (décors, accessoires), etc. Cette négociation pourra s'avérer particulièrement intéressante si vous attendez un nombre imposant de délégués ou de participants en provenance d'une même région ou d'un même pays.

La tenue de l'événement se rapproche et votre comité organisateur doit planifier la logistique de l'accueil des arrivants depuis les aéroports et gares de chemin de fer jusqu'aux hôtels et vice-versa. De mème le bon déroulement de la manifestation peut nécessiter la location de véhicules (autocars, minibus, camions, voitures) pour le transport des participants et des matériels (décors, accessoires, costumes, instruments de musique, etc.). Des contacts seront donc établis avec des compagnies de location de véhicules et possiblement avec des réseaux de guides-accompagnateurs multilingues.

Il faut également souligner l'aspect divertissement ou découverte touristique inhérent à toute manifestation nationale ou internationale de quelle que nature qu'elle soit. Afin d'offrir un service complet et personnalisé à vos participants, délégués et festivaliers, la disponibilité d'activités touristiques attrayantes - depuis la visite guidée de la ville ou de la région où a lieu l'événement jusqu'à des séjours pré- ou post-événement dans des régions limitrophes renommées pour leurs attraits touristiques et/ou de villégiature et/ou culturels - est un élément additionnel souvent apprécié. Voila bien de toute évidence un secteur d'activités relevant d'une agence de voyages. Vous pouvez tenter de pré-vendre ces activités touristiques ou encore vous 
assurer de leur disponibilité durant l'événement grâce â un comptoir-voyages facile d'accès, à proximité du quartier général de votre organisation, ou au mieux, combiner les deux possibilités: pré-vente et vente sur place durant l'événement.

Peut-étre n'envisagez-vous pas confier les tâches et les dossiers ci-haut énumérés à des spécialistes touristiques mais le but était bien de démontrer la diversité des contacts essentiels avec des partenaires touristiques, c'est-ă-dire dans quels secteurs spécifiques vous auriez interét à transiger avec des intervenants en tourisme et dans quelle mesure vous pourriez recourir aux services d'une agence de voyages qualifiée, vous déchargeant ainsi de la logistique technique.

Actuellement, à notre avis, ce sont les agences réceptives qui sont les mieux positionnées pour vous assurer tant une expertise qu'un soutien technique efficace. $\mathrm{Ce}$ type d'agences regroupe des professionnels de l'industrie du voyage, spécialisés dans l'accueil individuel ou de groupes étrangers en visite au Canada et au Québec. C'est un concept - le tourisme réceptif - encore jeune chez nous. Ces agences vendent notre destination sur les marchés étrangers, connaissent les ressources des grands centres que sont Montréal, Québec, Toronto, etc. et profient en général d'un volume de ventes suffisamment élevé leur permettant de négocier des prix abordables et compétitifs pour une foule de prestations de services. De plus, toute agence sćrieuse veillera à respecter le caractère, l'atmosphère et le type de services recherché par votre organisation. Par la, nous entendons qu "un congrès scientifique ou une réunion d'administrateurs n'ont pas le même esprit qu'un festival de théătre ou qu'un tournoi international de tennis où souvent décontraction et ambiance de fête sont à l'honneur.

\section{L'insertion de l'événement dans le réseau de distribution touristique}

Une manifestation d'envergure - artistique ou sportive - doit avoir lieu. Il est normal de vouloir, à l'occasion de tels événements, maximiser la venue de touristes sur les plans local, provincial, national ou international en vue d'assister ou prendre part à l'événement. Le Festival de Jazz de Montréal, le Festival d'été de Québec sont des exemples de réussite pour attirer les touristes en milieux urbains au Québec durant les chaleurs de juillet. Mais pour attirer un public toujours elargi et de ce fait, hors des cercles d'inconditionnels, il faut compter sur une stratégie de promotion dynamique. Nous avons vu au chapitre de l'aide technique les avenues de collaboration possibles avec des partenaires touristiques; qu'en est-il maintenant de cette collaboration afin d'accroitre la visibilité d'une manifestation à l'intérieur du réseau de distribution touristique?

Considérons d'abord les différents paliers gouvernementaux. Au fédéral, l'Office de tourisme du Canada, et au provincial, Tourisme Québec, publient annuellement des brochures touristiques à l'intention tant de leurs marchés intérieurs qu'extérieurs en plus d'organiser des foires où des acheteurs touristiques (grossistes en voyages, transporteurs, etc.) de toutes provenances, viennent se familiariser avec les produits touristiques canadiens et québécois. Toute manifestation d'envergure et toute fete populaire reliées à des patrimoines régionaux (historique, gastronomique ou autre) devraient figurer dans ces listes et répertoires. Il peut difficilement être question de participer à ces foires promotionnelles en raison des coûts élevés impliqués mais la visibilité - mẻme minimale - peut amener des acheteurs étrangers à s'intéresser à Montréal ou Québec plutốt qu'à Amsterdam et Bruxelles.

Depuis peu, existe, au fédéral, un ministre d'Etat au Tourisme (distinct de celui de la Petite Entreprise) et au Québec, en 1984, un ministère du Tourisme dissocié du ministère de l'Industrie et du Commerce. Le tourisme commence donc à être considéré comme révélant un potentiel économique important alors que l'industrie touristique - canadienne et québecooise - sort d'une période de crise. Le début des années ' 80 a vu chuter le nombre de touristes étrangers entrant au Canada et augmenter la balance déficitaire du compte voyages mais l'année 1985 a connu des sommets inégalés depuis 1967 et l'Exposition Universelle de Montréal.

Montréal, Québec, le Canada entier cherchent depuis quelques années à mieux définir ou redefinir leur vocation touristique. Le tourisme d'événements est-il déjà en train de s'implanter et de servir de levier pour insuffler un nouveau dynamisme à l'industrie touristique? Dans le cadre de l'entente auxiliaire Canada-Québec sur le tourisme, signée au début de 1985 , certains événements majeurs - artistiques ou sportifs - peuvent bénéficier d'une attention spéciale de nos gouvernements et de subventions touchant directement aux campagnes de publicité (ou plan-médias). Il est évident que ces subventions, lorsqu'elles sont accordées, doivent être utilisées horsQuébec et/ou hors-Canada pour des activités publicitaires et promotionnelles visant à attirer des touristes standards ou des publics spécialisés.

D'autres clés sont également à connaittre et a utiliser pour rejoindre à peu de frais un plus grand bassin de population et ce, particuliêrement au niveau provincial et régional: les kiosques et Maisons du tourisme (ex: la Maison du tourisme du Québec à la Place Ville-Marie à Montréal) ainsi que les associations touristiques régionales. implantées comme leur nom l'indique, aux quatre coins de la Belle Province. Il faut vous assurer de la disponibilité en ces endroits de vos outils promotionnels (programmes, affiches, épinglettes, etc.) et de figurer dans les brochures annuelles de votre association touristique. Certaines associations touristiques (A.T.R.) proposent même ou font mettre sur pied des forfaits touristico-culturels en saison estivale. L'exemple classique est une visite touristique combinée à une soirée dans un thêtre d'été; plusieurs régions, done plusieurs intervenants touristiques et artistiques, connaissent des résultats impressionnants avec ce type de formule à forfait.

Existent également les bureaux de tourisme et des congrès dans les grandes localités du Québec (Sherbrooke, Trois-Rivières, Chicoutimi, Jonquière, etc.) qui peuvent aider a la promotion de toute manifestation et ce, de façon relativement peu dispendieuse si ce n'est tout à fait gratuite ${ }^{(2)}$. Il faut cependant nous attarder à deux cas particuliers: Québec et Montréal dont les offices de tourisme et des congrès jouent le rỏle d'une association touristique régionale. À Québec, le Bureau du tourisme et des congrès est rattaché à la Communauté urbaine de Québec; à Montréal, son pendant travaille en étroite collaboration avec le Palais des congrès de Montréal et avec CIDEM (Commission d'initiative et de développement économique de Montréal) - secteur Tourisme. Encore une fois, il vous faut être connu de ces organismes qui peuvent vous publiciser lors de foires promotionnelles (ex.: Salon Vacances Québec), vous aider dans certains besoins logistiques mais surtout vous aider à diffuser la tenue de votre événement en Ontario et aux États-Unis par le biais de campagnes publicitaires dans les grands médias et via d'imposants réseaux de chroniqueurs spécialement attachés aux sections Travel and Tourism des grands quotidiens ou magazines canadiens et américains.

Se faire connaittre est déjà un premier pas, mais il semble manquer des éléments de commercialisation pour bien assurer la diffusion et la promotion d'événements. On n'attire pas uniquement les touristes par des annonces dans les grands quotidiens. Les agences et réseaux d'agences de voyages sont un possible réseau de distribution touristique pour vos événements.

\section{Avec qui collaborer?}

Les agences de voyages détaillantes? Les réseaux d'agences détaillantes? Les grossistes en voyages? Les agences de tourisme réceptif? Laquelle de ces alternatives peut répondre le plus adéquatement à vos besoins?

Une agence de voyages détaillante pourrait négocier vos billets d'avion, horaires 
et itinéraires, tant pour vos déplacements durant la période d'organisation que ceux de vos invités et participants mais son réseau de distribution est en général limité à sa clientèle habituelle: donc faible utilité quant à la commercialisation extensive de votre produit. Les regroupements d'agences vous permettent déjà d'accéder à des clientèles-cibles élargies. vu le pouvoir d'achat accru de ces regroupements et les budgets supérieurs consentis à la publicité. Si vous êtes organisateur d'un événement important se déroulant en région et qui vise une clientèle québécoise, provinciale, la possibilité de vous associer à un réseau d'agences présent presque partout au Québec est intéressante, mais une question reste de taille. Atteindrez-vous la clientèle que vous visez et cette clientèle est-elle acheteuse des services offerts par un tel regroupement? Majoritairement, les gens recourent aux agences de voyages pour les déplacements éloignés au Canada et à l'étranger; rarement en ce qui concerne les séjours de vacances au Québec et en Ontarió. L'automobile est le moyen de transport le plus fréquemment utilisé et on couche souvent chez des parents ou des amis.

Quant aux grossistes en voyages, ils n'ont pas souvent l'habitude d'offrir à leurs clients des produits culturels et novateurs. Chaque grossiste se concentre en général sur un petit nombre de destinations bien spécifiques: le soleil à l'étranger, le ski en hiver au Canada et en Europe, des programmes de tourisme standards - formule circuits accompagnés - le plus souvent axés sur les attraits de villes touristiques majeures au niveau commercial. Qu'advient-il alors si votre manifestation a une portée nord-américaine ou internationale? Il faut se l'avouer: le principal but recherché par les promoteurs culturels au niveau de la promotion est d'accroitre le nombre d'entrées aux guichets en diversifiant la publicité aux coûts les plus bas. Spécialement dans le domaine culturel au Canada (Québec), les budgets alloués à la publicité d'événements majeurs sont souvent minces. Donc, si votre événement lorgne vers l'international et que vous songez à collaborer avec une entreprise touristique, la maison retenue doit être susceptible de générer des clientèles venant de l'étranger et à peu de frais promotionnels.

Nous avons déjà brièvement parlé des agences réceptives. Des événements majeurs et des fétes populaires à saveur québécoise peuvent ici devenir des attraits additionnels et originaux, servant à mieux vendre la destination Québec à l'étranger

Les agences réceptives confectionnent des forfaits selon une formule rable $d$ 'horte. c'est-à-dire des circuits standards que l'on peut déjà acheter tout constitués de l'étran. ger, ou à la carte, c'est-à-dire des forfaits exclusifs et sur mesure, préparés avec grand soin sur demande d'agences de voya- ges étrangères pour groupes déjà constitués ou en voie de formation. En général, elles veillent à la promotion de la destination canadienne par le biais de brochures et catalogues, annuels ou semi-annuels, avec forfaits pré-établis ou encore par une brochure énumérant uniquement les services en mesure d'être offerts. Chaque agence réceptive développe son propre réseau de distribution à l'étranger, par exemple en devenant le partenaire exclusif de grossistes européens ou américains au Canada; ou encore, en adhérant à un réseau réceptif international (ex.: International Travel Partners). Via ces canaux, votre événement pourrait accéder à une visibilitế promotionnelle intéressante à l'étranger. D'autres agences réceptives privilégient des marchés spécifiques - tourisme étudiant, clientèles européennes, etc.-, des types de voyages spécifiques (congrès, voyagesmotivation, circuits strictement touristiques, etc.) mais jusqu'à maintenant, peu se sont impliquées dans la promotion touristique d'évênements majeurs si ce n'est à l'occasion d'une demande ponctuelle, originant de l'étranger.

C'est que les événements majeurs comportent pour l'industrie touristique et pour les promoteurs eux-mêmes de sérieuses inconnues en termes de rentabilité économique. Il faut ici méditer sur la différence de taille qui existe entre la tenue d'un congrès d'une association $\mathrm{X}$ et celle du festival $\mathrm{Y}$, par exemple. Dans le cadre d'un congrès, quoique là aussi la marge d'erreur peut se révéler, à l'expérience, assez élevée, on peut davantage s'appuyer sur des éléments statistiques connus: nombre total de membres dans la dite association; assistances aux congrès tenus par le passé; attraction du fait que les villes où se déroulent les congrès de l'association varient; importance de la connotation travail-vacances dans l'esprit du congressiste dont l'association défraiera en bonne partie les frais de séjour, etc. Dans le cadre de manifestations artistiques même prestigieuses, vous avouerez qu'il est plutót difficile de répertorier le nombre d'amateurs d'opéra aux Etats-Unis, de fons de cinéma moderne en Europe de l'Ouest, etc. Malgré le caractère poussé de cette comparaison, vous devez être préparé à ces constatations réalistes et pragmatiques si vous envisagez de transiger avec des partenaires de l'industrie touristique.

L'estimation des visiteurs-participants que vous pourrez fournir peut, à la base, être décisive. N'en demeure pas moins possible et souhaitable une collaboration entre partenaires touristiques et artistiques (sportifs). C'est en précisant, en cernant le mieux possible les clientèles cibles visées que vous pourrez économiser temps et energie de même qu'identifier l'agence qui correspond le mieux à vos attentes. Quelle est la portée de votre écénement? Votre clientèle est-elle grand public ou spécialisée: gens d'art, étudiants, groupes ethnolinguistiques, etc.?
L'aide à la promotion ou à la visibilité de votre évênement se concrétisera généralement par la création de forfaits spécifiques à votre événement. Elle pourrait nécessiter des mises de fonds au point de départ. Ce volet est délicat par plusieurs aspects. Vous confiez à une agence touristique le mandat de vous représenter à l'étranger. II sera donc important de vérifier rapidement si votre partenaire comprend ce que vous lui apportez, en termes d'image et de contenu. Il sera aussi question d'échéancier de production selon le calendrier de parution des brochures de l'agence choisie ou encore des modalités de production d'une brochure commune - événement/agence - spécifique à votre manifestation. Dès le début de la collaboration. il sera essentiel de connaître le moment où la programmation officielle de l'événement sera disponible car cela pourrait influer sur la stratégie promotionnelle à retenir.

Autre élément-clé dans la mise en marché d'un événement d'envergure: le réseau de billetterie ou l'accessibilité que vous favorisez pour votre manifestation. Cela aura une incidence majeure sur les clientèles que vous pourrez rejoindre et attirer. Opterezvous pour une billetterie entièrement manuelle et centralisée dans la ville de l'evénement ou pour un système information et décentralisế? Si vous envisagez collaborer avec une agence, il vous faudra garantir à cette dernière la disponibilité de billets à ses clients potentiels. Traiter de la billetterie nous amène à parler de "prévente", cette période cruciale et souvent déterminante dans le succès d'un événement artistique ou sportif. Il faut la bien planifier, le plus longtemps à l'avance possible. Si vous songez à une période de prévente d'environ 4 à 6 semaines, la programmation définitive devra être minimalement finalisée trois mois avant l'ouverture de l'événement. Et nous devons penser à cinq ou six mois à l'avance si vous comptez effectuer un "blitz" promotionnel à l'étranger.

Voila bien des êléments à considérer avant de $s^{*}$ engager avec des partenaires touristiques.... peut-être une collaboration ne pourra être sur-le-champ envisagée... Alors, à quoi bon faire toutes ces démarches? C'est que ces rencontres sur le terrain amènent une sensibilisation des deux secteurs, amoindrissent l'ignorance des ré̉alités vécues par les intervenants touristiques et les agents culturels et sportifs. Malgré l'expérience souvent fort valable des agences, malgré la collaboration qui va grandissant avec les instances gouvernemen= tales, nous n'en sommes encore qu'aux premiers balbutiements de ce qui représente actuellement un grand defi: l'insertion des événements majeurs comme de véritables attractions dans le réseau de distribution touristique au Québec. 